\title{
Bone Cell Autophagy Is Regulated by Environmental Factors
}

\author{
Adam M. Zahm ${ }^{a} \quad$ Jolene Bohensky ${ }^{b}$ Christopher S. Adams ${ }^{c} \quad$ Irving M. Shapiro $^{b}$ \\ Vickram Srinivas ${ }^{b}$

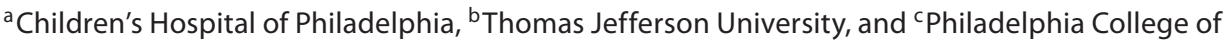 \\ Osteopathic Medicine, Philadelphia, Pa., USA
}

\section{Key Words}

Bone cells $\cdot$ Hypoxia $\cdot$ Autophagy $\cdot$ Autophagic flux $\cdot$

Hypoxia inducible factor 1

\begin{abstract}
The goal of this investigation was to ascertain whether bone cells undergo autophagy and to determine if this process is regulated by environmental factors. We showed that osteocytes in both murine and human cortical bone display a punctuate distribution of microtubule-associated protein light chain 3, indicative of autophagy. In addition, we noted a basal level of autophagy in preosteocyte-like murine long bone-derived osteocytic (MLO)-A5 cells. Autophagy was upregulated following nutrient deprivation and hypoxic culture, stress conditions that osteocytes encounter in vivo. Furthermore, in response to calcium stress, the transcription factor hypoxia inducible factor 1 regulated MLO-A5 autophagy. Finally, we showed that the more differentiated MLO-Y4 osteocyte-like cells exhibited a significant basal autophagic flux. Based on these findings, we suggest that raising the level of autophagic flux is a mechanism by which differentiated bone cells survive in a stressful environment.
\end{abstract}

Copyright $\odot 2011$ S. Karger AG, Basel

Vickram Srinivas is deceased.

\section{Introduction}

Among the many systems required for survival is the evolutionarily conserved autophagosome-lysosomal (autophagy) pathway. Bulk macroautophagy not only plays a housekeeping role in eliminating old organelles and misfolded and damaged molecules but it is also required in times of metabolic need. Thus, during acute hypoxia and loss of nutrient supply, autophagy promotes the breakdown of complex molecules that are then used for metabolic, energetic, and biosynthetic purposes [Wellen and Thompson, 2010]. In this context we, and others, recently showed that autophagy and autophagic flux is stimulated by hypoxia inducible factor (HIF)-1 [Bohensky et al., 2009]. The goal of the current investigation is to examine the induction of autophagic flux in bone cells. We report for the first time that human osteocytes undergo autophagy in vivo. In addition, using preosteocyte and osteocytic cell lines, we show that the autophagic response is dependent on the expression of HIF-1.

\section{Abbreviations used in this paper}

HIF hypoxia inducible factor

LC3-I microtubule-associated protein light chain 3-I

LC3-II microtubule-associated protein light chain 3-II

MLO murine long bone-derived osteoblastic/osteocytic cell lines

\section{KARGER}

๑๐ 2011 S. Karger AG, Basel

Fax +4161306 1234

E-Mail karger@karger.ch

www.karger.com
Accessible online at: www.karger.com/cto
Dr. Irving M. Shapiro

Department of Orthopaedic Surgery, Thomas Jefferson University

501 Curtis Building, 1015 Walnut Street, Suite 501

Philadelphia, PA 19107 (USA)

Tel. +1 215955 7217, E-Mail irving.shapiro@jefferson.edu 
Fig. 1. LC3 is expressed in cortical bone osteocytes. a Longitudinal section of rat tibial cortical bone. Osteocytes can be seen (inset) exhibiting a punctate LC3 distribution indicative of autophagy. b Transverse section of a femoral condyle. Note the punctate LC3 distribution (inset).
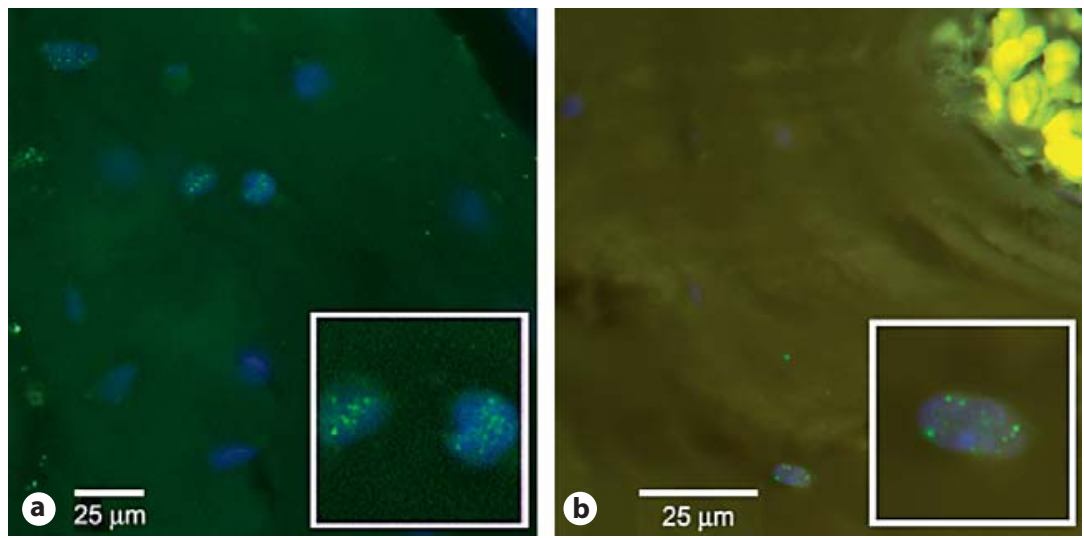

\section{Materials and Methods}

Bone Cell Culture

Murine long bone-derived osteocytic (MLO) A5 and Y4 cell lines (gifts from Dr. Lynda Bonewald) were cultured in an inVIVO $_{2}$ hypoxia workstation [Kato et al., 2001]. For some experiments, cells were treated with $200 \mathrm{nM}$ bafilomycin A, $25 \mathrm{nM}$ rapamycin, or $1 \mu \mathrm{M}$ thapsigargin for 5 or $6 \mathrm{~h}$.

In vivo Immunolocalization of Microtubule-Associated

Protein Light Chain 3 (LC3) in Bone

Ten-month-old Wistar rats were sacrificed using IACUC-approved methods, while human femoral condyles following knee arthroplasty were collected with IRB approval. Bones were fixed, demineralized, and sectioned. Following antigen retrieval and treatment with Triton X-100, they were blocked, incubated with rabbit anti-MAP1LC3b (LC3) (1:50), and treated with goat antirabbit IgG-488 (Invitrogen, Carlsbad, Calif., USA) (1:200).

In vitro Immunolocalization of LC 3 in MLO Cells

Cultures were fixed, permeabilized, and blocked in 10\% FBS. They were incubated with rabbit anti-MAP1LC3 (1:500) and treated with goat anti-rabbit IgG-488 (1:200).

Electron Microscopic Evaluation of Autophagy

Cells were fixed, postfixed with $\mathrm{OsO}_{4}$, and stained in uranyl acetate. They were then embedded in Epon-Araldite resin and ultrathin sections were counterstained with $0.3 \%$ lead citrate.

Western Blot Analysis

Cells were processed for Western blot analysis as described previously [Bohensky et al., 2009].

siRNA-Mediated Suppression of HIF-1 $\alpha$

The nucleotide sequence 5' GTCCAGAGTCACTGGGACT (nucleotides 1,438-1,457; mouse HIF-1 $\alpha$ mRNA) was silenced by RNA interference, and permanent cell lines were selected using puromycin as described previously [Zahm et al., 2008]. A cell line with the reversed sequence was used as the control. Expression levels were verified by RT-PCR and Western blot analysis [Bohensky et al., 2009].

\section{Results}

\section{Osteocytes Display an Autophagic Phenotype}

Immunohistological examination of longitudinal sections of a rat tibia showed that LC3 is distributed in a punctate pattern in osteocytes (fig. 1a). Similarly, in transverse sections of the human femoral condyle punctate LC3 staining was seen in osteocytes, suggesting that these cells are autophagic (fig. 1b). Particulate LC3 staining was not observed in osteoblasts or osteoclasts on bone surfaces or in osteocytic processes.

\section{MLO-A5 Preosteocytes Exhibit an Inducible Basal Autophagic Flux}

Figure $2 \mathrm{a}$ and $\mathrm{b}$ shows that MLO-A5 cells generate the LC3-II isoform, indicative of its association with the autophagosome. Furthermore, inhibition of the lysosomal V-ATPase with bafilomycin A1 caused accumulation of the LC3-II isoform suggesting that these cells have an active autophagic flux. To determine whether this flux could be further induced, cells were treated with the autophagic stimulator rapamycin. Figure $2 \mathrm{c}$ and $\mathrm{d}$ shows that rapamycin increased LC3-II levels. Moreover, when stressed by serum withdrawal, there was an increase in LC3-II levels (fig. 2e, f).

\section{Anoxia Significantly Induces Autophagy in \\ Preosteocytes}

Figure $3 \mathrm{a}$ and $\mathrm{b}$ shows that while basal autophagy was observed under normoxic conditions $\left(20 \% \mathrm{O}_{2}\right)$, there was a slight increase in LC3-II level under hypoxic conditions $\left(2 \% \mathrm{O}_{2}\right)$ and a significant increase in LC3-II levels in anoxia $\left(0 \% \mathrm{O}_{2}\right)$. 


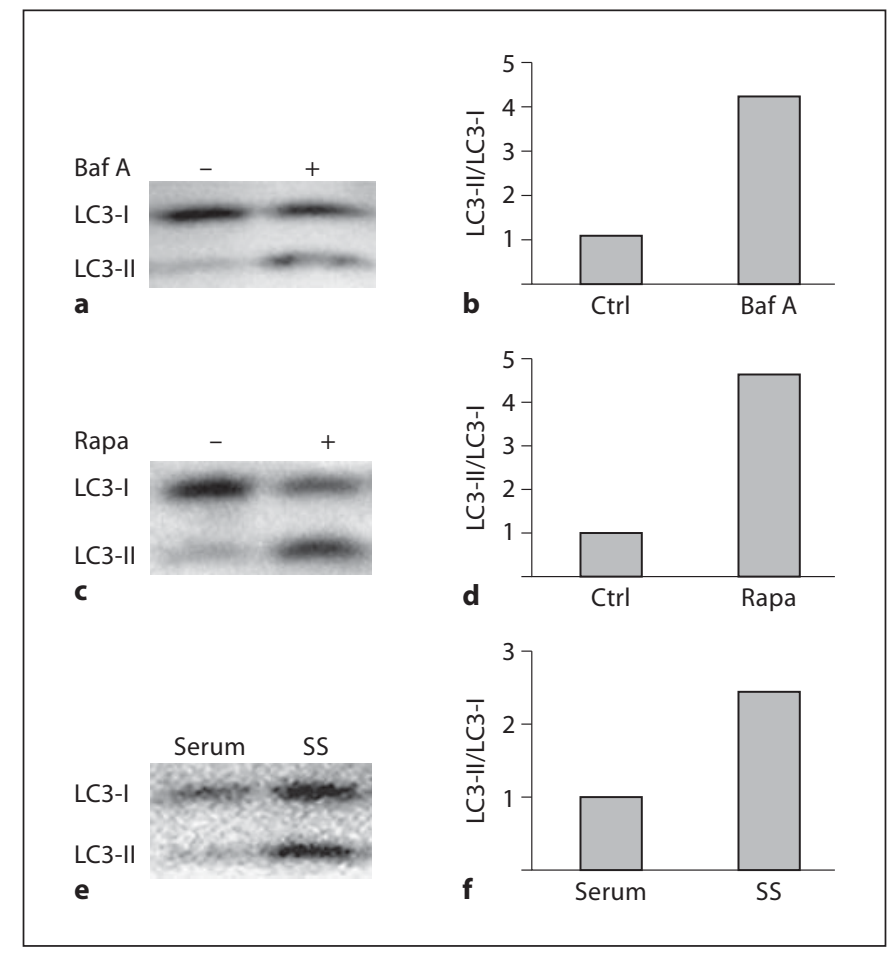

Fig. 2. MLO-A5 preosteocytes display an inducible autophagic flux. a, b Increase in LC3-II expression following bafilomycin A1 (Baf A) treatment. Cells were grown in the absence (-) or presence $(+)$ of Baf $\mathrm{A}$ for $5 \mathrm{~h}$ and lysate LC3 expression was determined by Western blot analysis. Note the accumulation of the LC3-II isoform in cells treated with Baf A. c, d Induction of autophagy by rapamycin (Rapa). MLO-A5 cells were grown in the absence or presence of Rapa for $6 \mathrm{~h}$ and lysates were examined for LC3 expression by Western blot. The increase in LC3-II upon Rapa treatment is indicative of the induction of autophagy. e, f Serum starvation (SS)-mediated induction of autophagy. There was a significant increase in LC3-II levels in SS cells.

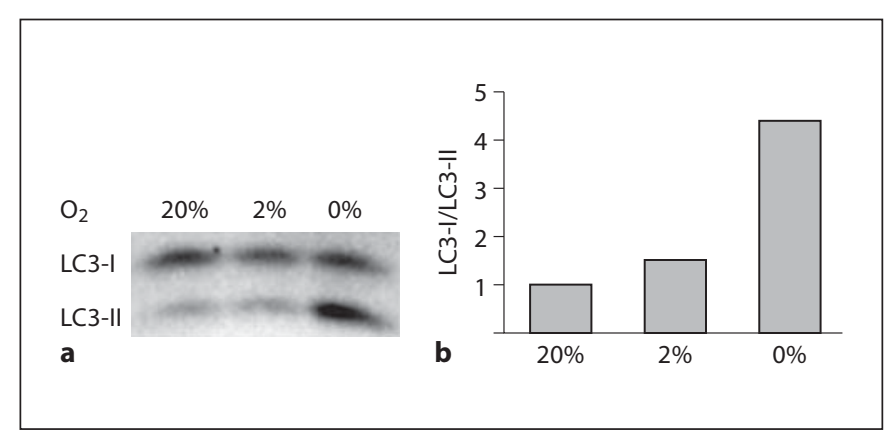

Fig. 3. Anoxia promotes the induction of autophagy. Cells were grown in normoxia $\left(20 \% \mathrm{O}_{2}\right)$, hypoxia $\left(2 \% \mathrm{O}_{2}\right)$, or anoxia $\left(0 \% \mathrm{O}_{2}\right)$ for $6 \mathrm{~h}$ and LC3 expression was examined by Western blot analysis. There was an increase in the LC3-II isoform in the anoxic cells. The LC3-II/LC3-I ratio of the $20 \% \mathrm{O}_{2}$ culture is corrected to a value of 1 .

\section{Thapsigargin Induces Autophagy and HIF-1 $\alpha$ in} MLO-A5 Cells

We next treated MLO-A5 cells with thapsigargin, an inhibitor of the sarco/endoplasmic reticulum $\mathrm{Ca}^{2+}$ ATPase pumps that regulate cytoplasmic calcium levels. Thapsigargin treatment resulted in an increase in autophagy as evidenced by an increase in LC3-II levels (fig. 4a, b) and a punctate redistribution of LC3 (fig. 4c). We next examined HIF- $1 \alpha$ expression following thapsigargin treatment. This drug caused a robust increase in HIF- $1 \alpha$ protein levels at $20 \% \mathrm{O}_{2}$ (fig. $4 \mathrm{~d}$ ).

\section{HIF-1 $\alpha$ Is Required for Thapsigargin-Induced}

Autophagy in MLO-A5 Preosteocytes

In (inverted) control cells, thapsigargin treatment resulted in the accumulation of LC3-II, indicating an active flux (fig. 4e, f). However, when HIF-1 $\alpha$ was silenced, no accumulation of LC3-II was observed, indicating that this transcription factor is required for the induction of autophagic flux following calcium stress.

\section{MLO-Y4 Cells Exhibit Significant Basal Autophagic Flux}

There was a significant expression of LC3-II in MLOY4 osteocytes, indicating basal autophagy (fig. 5a, b). We confirmed the presence of autophagosomes by TEM. Figure $5 \mathrm{c}$ shows that few autophagosomes were formed in the MLO-A5 cells; double membrane-lined vesicles as well as autophagic isolation membranes were visible in the MLO-Y4 osteocyte-like cells (fig. 5d).

\section{Discussion}

The goal of this investigation was to determine whether bone cells undergo autophagy and to ascertain if this process is regulated by environmental factors. In both rat and human cortical bone, many osteocytes exhibited a punctate distribution of LC3, a hallmark of autophagosome formation. Particulate LC3 fluorescence was not observed in osteoblasts or bone-lining cells. The autophagic osteocytes were most often seen at a distance from the haversian canal. However, since a simple gradient in autophagic cells was not evident, it must be presumed that multiple factors regulate the autophagic response.

Little is known concerning the induction of autophagic flux in bone cells in culture. In the unstimulated state, the less differentiated MLO-A5 cells expressed a low basal level of LC3-II together with the presence of some au- 
Fig. 4. Thapsigargin (Thap)-mediated induction of autophagy in MLO-A5 cells is dependent on HIF-1. a, b Induction of autophagy by Thap. Following Thap treatment for $6 \mathrm{~h}$, lysates were assessed for LC3 expression by Western blot. There was a robust increase in LC3-II expression in Thap-treated cells. c LC3 immunofluorescence analysis following Thap treatment. Note the distinct punctate pattern of LC3 expression (arrows) in the treated cells. d Increased HIF-1 $\alpha$ expression in Thaptreated cells. Following treatment with Thap for $6 \mathrm{~h}, \mathrm{HIF}-1 \alpha$ expression was assessed by Western blot analysis. Note the increased expression of HIF- $1 \alpha$. e, $\mathbf{f}$ Thapmediated induction of autophagy is dependent on HIF- $1 \alpha$. HIF- $1 \alpha$ silenced and inverted control (Inv Ctrl) cells were treated with Thap for $6 \mathrm{~h}$. Whole cell lysates were assessed for LC3 expression by Western blot. There was an increase in LC3-II expression in the Thap-treated control cells. When HIF-1 was silenced, no change in LC3II levels was observed.

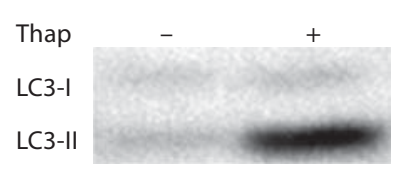

a

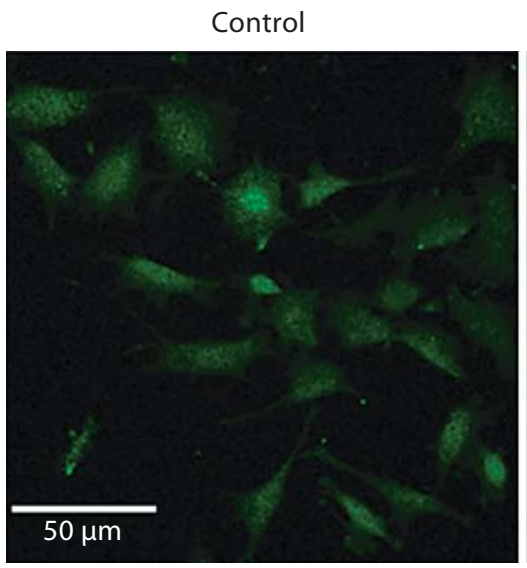

C

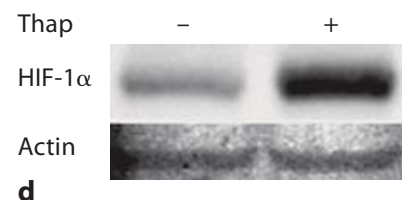

d

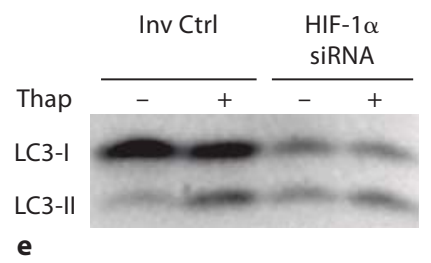

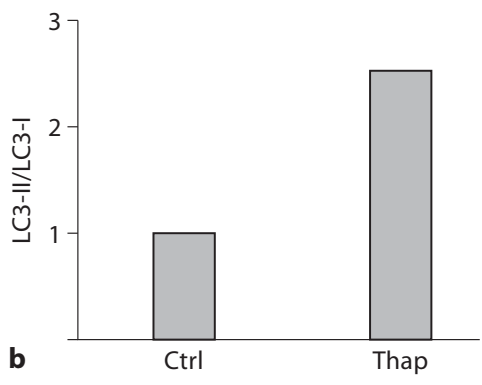

Thap
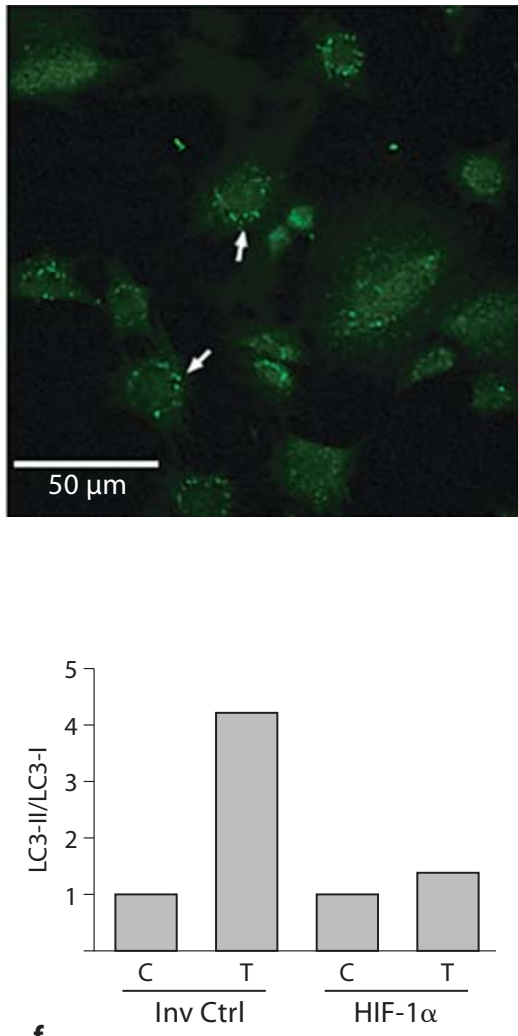

tophagic vacuoles and isolation membranes. In contrast, the more differentiated MLO-Y4 osteocyte-like cells exhibited elevated levels of LC3-II together with a considerable number of double membrane autophagosomes as well as defined isolation membranes. These in vitro studies lend support to the in vivo finding that, with differentiation, bone cells express a robust autophagic state.

We examined autophagy in terms of microenvironmental factors that influence preosteocyte metabolism. Predictably, in serum-free medium, there was an increase in autophagosome formation; bafilomycin inhibition of vesicle acidification caused an accumulation in LC3-II levels, suggesting an active autophagic flux [Iwai-Kanai et al., 2008]. Rapamycin, a stimulator of autophagy, caused a marked increase in LC3-II levels [Yu et al., 2010]. These observations provide evidence that cells can respond rapidly to external conditions by regulating autophagic flux. That many osteocytes in vivo are in an elevated autophagic state would suggest that this pathway is operative in cells removed from growth factors and nutrients.

The observation that very low $\mathrm{pO}_{2}$ promoted an autophagic state lends clear support to the notion that envi- 
Fig. 5. MLO-Y4 osteocytes display elevated basal autophagic flux. a, b LC3-II expression in MLO-Y4 and MLO-A5 cells. LC3II expression was assessed by Western blot analysis. Note that in MLO-Y4 cells LC3-II expression is more robust than in MLOA5 cells. TEM analysis of autophagy in MLO-A5 (c) and MLO-Y4 (d) cells. Autophagosomes and isolation membranes are present in MLO-A5 preosteocytes (arrows; c). Double membrane autophagosomes and isolation membranes were observed in MLO-Y4 osteocytes (arrows; d).

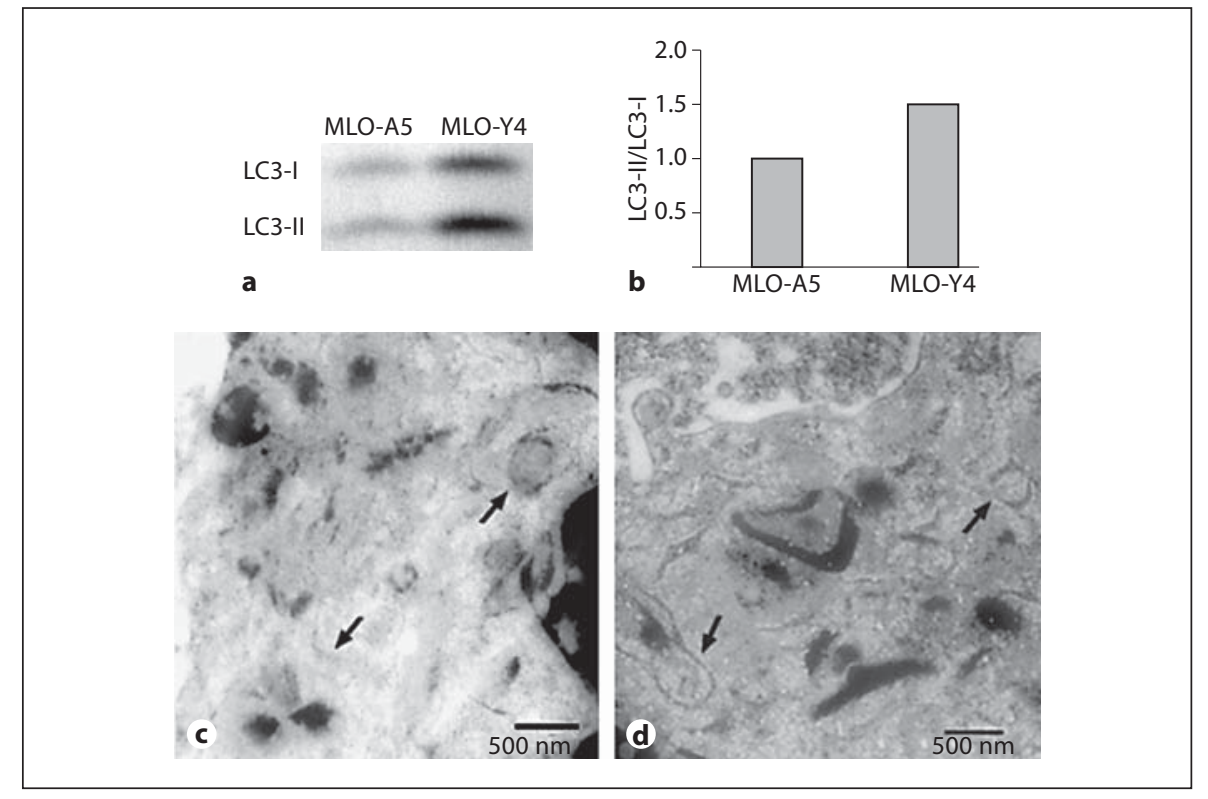

ronmental factors regulate the survival of bone cells. We also noted that changes in ER calcium flux, linked to skeletal cell mechanotransduction and apoptosis, modulated autophagy. Inhibition of the calcium ATPase with thapsigargin resulted in an increase in the formation of punctate, LC3-II-labeled autophagosomes, a result which is in contrast to the recent report that UPR causes a block in flux [Rzymski et al., 2009]. Thus, changes in intracellular calcium due to high functional loading, osteogenesis imperfecta, or metabolic diseases could ultimately be linked to overstimulation of the autophagic flux, resulting in premature cell death.

Surprisingly, thapsigargin treatment increased HIF$1 \alpha$ levels. In addition, HIF- $1 \alpha$ was found to be required for thapsigargin-induced autophagy. This new finding accords with our earlier study showing that HIF-1 $\alpha$ promotes autophagy in chondrocytes [Bohensky et al., 2009]. Thus, in addition to regulating the maturation and mineralization of bone cells, HIF-1 regulates the induction of bone cell autophagy [Zahm et al., 2008].

\section{Acknowledgements}

This study was supported by NIH grants RO1 DE010875, RO1 DE013319, RO1 DE016383, and T32 AR052273, and by a grant from the Commonwealth of Pennsylvania - Department of Health (SAP No. 4100026302).

\section{References}

Bohensky, J., S.P. Terkhorn, T.A. Freeman, C.S. Adams, J.A. Garcia, I.M. Shapiro, V. Srinivas (2009) Regulation of autophagy in human and murine cartilage: hypoxia-inducible factor 2 suppresses chondrocyte autophagy. Arthritis Rheum 60: 1406-1415.

-Iwai-Kanai, E., H. Yuan, C. Huang, M.R. Sayen, C.N. Perry-Garza, L. Kim, R.A. Gottlieb (2008) A method to measure cardiac autophagic flux in vivo. Autophagy 4: 322-329.
Kato, Y., A. Boskey, L. Spevak, M. Dallas, M. Hori, L.F. Bonewald (2001) Establishment of an osteoid preosteocyte-like cell MLO-A5 that spontaneously mineralizes in culture. J Bone Miner Res 16: 1622-1633.

- Rzymski, T., M. Milani, D.C. Singleton, A.L. Harris (2009) Role of ATF4 in regulation of autophagy and resistance to drugs and hypoxia. Cell Cycle 8: 3838-3847. PMID: 19887912.

Wellen, K.E., C.B. Thompson (2010) Cellular metabolic stress: considering how cells respond to nutrient excess. Mol Cell 40: 323-332.
Yu, L., C.K. McPhee, L. Zheng, G.A. Mardones, Y. Rong, J. Peng, N. Mi, Y. Zhao, Z. Liu, F. Wan, D.W. Hailey, V. Oorschot, J. Klumperman, E.H. Baehrecke, M.J. Lenardo (2010) Termination of autophagy and reformation of lysosomes regulated by mTOR. Nature 465: 942-946.

Zahm, A.M., M.A. Bucaro, V. Srinivas, I.M. Shapiro, C.S. Adams (2008) Oxygen tension regulates preosteocyte maturation and mineralization. Bone 43: 25-31. 